Alma Mater Studiorum - Università di Bologna DEPARTMENT OF ECONOMICS

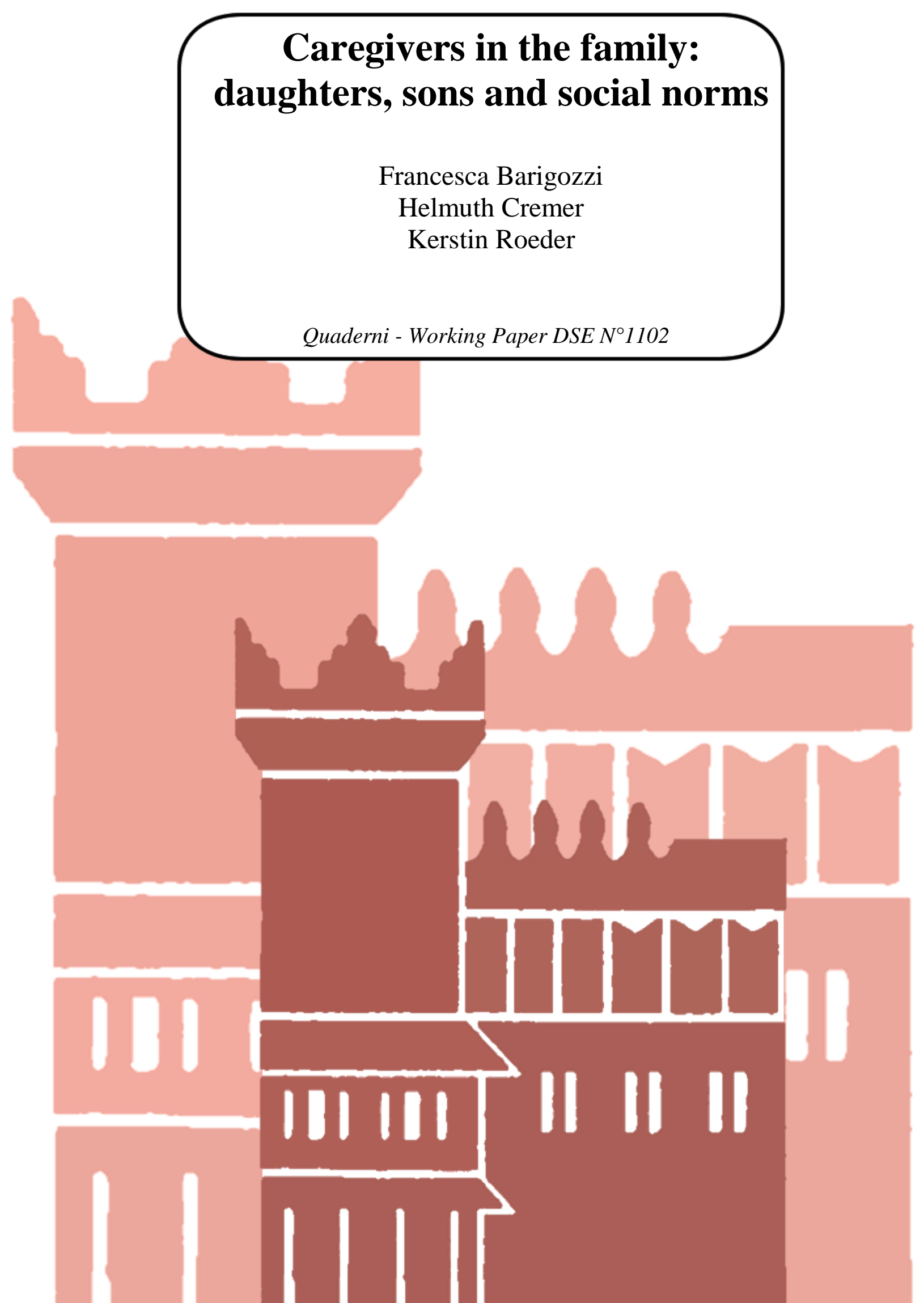




\title{
Caregivers in the family: daughters, sons and social norms*
}

\author{
Francesca Barigozzi ${ }^{\dagger} \quad$ Helmuth Cremer ${ }^{\ddagger} \quad$ Kerstin Roeder $^{\S}$
}

April 2017, revised June 2017

\begin{abstract}
Daughters are the principal caregivers of their dependent parents. In this paper, we study long-term care (LTC) choices by bargaining families with mixed- or same-gender siblings. LTC care can be provided either informally by children, or formally at home or in an institution. A social norm implies that daughters suffer a psychological cost when they provide less informal care than the average child. We show that the laissez-faire (LF) and the utilitarian first-best (FB) differ for two reasons. First, because informal care imposes a negative externality on daughters via the social norm, too much informal care is provided in LF. Second, the weights children and parents have in the family bargaining problem might differ in general from their weights in social welfare. We show that the FB allocation can be achieved through a system of subsidies on formal home and institutional care. Except when children and parents have equal bargaining weights these subsidies are gender-specific and reflect Pigouvian as well as "paternalistic" considerations.
\end{abstract}

$\boldsymbol{J E} \boldsymbol{L}$-Classification: D13, H23, H31, I19

Keywords: Social norms, formal and informal LTC, daughters, sons

*Financial support from the Chaire "Marché des risques et creation de valeur" of the FdR/SCOR is gratefully acknowledged. This paper has been presented at the EHEW in Oslo. We thank all the participants and particularly our discussant Tor Iversen for their comments.

${ }^{\dagger}$ University of Bologna, Italy, Email: francesca.barigozzi@unibo.it.

${ }^{\ddagger}$ Toulouse School of Economics, France, Email: helmuth.cremer@tse-fr.eu.

${ }^{\S}$ University of Augsburg, Germany, Email: kerstin.roeder@wiwi.uni-augsburg.de. 


\section{Introduction}

The provision of adequate long-term care (LTC) to the dependent elderly represents a major challenge faced by all developed countries. While this problem is already looming right now, it will become even more pressing during the decades to come because of population aging. Elderly people who are affected by cognitive diseases, like Altzheimer's or other forms of dementia, or by motoric problems due to ALS or Parkinson's disease need assistance with their daily activities. These LTC services can be delivered either formally at home or in institutions, or informally by family members. LTC is not covered by health insurance and only a few countries have specific systems of adequate LTC insurance coverage. As a result most LTC is currently provided informally either by spouses when still alive or, more significantly, by a person's children. ${ }^{1}$

Bott et al. (2017) in a recent article on dementia and informal care in the US argue that "The best long-term care insurance is a conscientious daughter". Indeed, among adult children taking care of their old parents, daughters typically provide more informal care than sons (Arber and Ginn, 1990; Bracke et al., 2008; Haberkern and Szydlik, 2010; Schmid et al., 2012; Tolkacheva et al., 2014). Coward and Dwyer (1990), for instance, consider the 1982 US National Long-Term Care Survey and the National Survey of Informal Caregivers and find that: "within all sibling network categories, daughters were more likely than sons to be providing care to an impaired parent [...]". Jakobsson et al. (2016) show that, when the rationing of formal home care is guided by "statistical discrimination", the gender gap in informal care provision is even exacerbated. In particular, the authors document how, in Norway, impaired old people receive less formal care when they have a daughter. This is because, when assigning formal LTC to citizens in need, managers take into account a patient's family situation and the fact that daughters typically provide more informal care. Hence, the gender gap in care provision is even self-reinforcing since daughters will have to compensate the lower

\footnotetext{
${ }^{1}$ Norton (2000) estimates the share of informal care at about $2 / 3$ of total LTC.
} 
amount of formal care received by their parents with a larger provision of informal care.

This paper deals with the gender gap in care provision and analyzes the choices within the family which contribute to its emergence. Our explanation is based on two factors. First, sons and daughters have unequal job market opportunities which determine their opportunity cost of providing care. Second, there is a social norm according to which society expects daughters to be the main caregivers of their parents and which imposes a utility cost on daughters who deviate from this norm. Gender differences in wages are well documented and continue to exist in all OECD countries, where women with a median wage earn on average $15 \%$ percent less than their male counterpart (see for instance, O’Neill, 2003; Fortin, 2005; Blau and Kahn, 2006). The role of social norms is empirically more difficult to assess; they represent by their very nature a less tangible concept than opportunity costs. Haberkern and Szydlik (2010) find that the extent to which providing informal care to needy family member is considered a moral obligation varies between countries. They show that of those aged $65+$ in the northern countries interviewed by SHARE believed that the state should bear the primary responsibility for LTC, while in Mediterranean countries, the majority believed that the family should mainly be responsible. Further, they show that where the consensus that care is a family matter is strongest, the share of informal care provided by daughters is also largest. On the other hand, institutions and social norms did not influence care relationships with sons. In a similar vein, Kotsadam (2011) finds that there is link between gendered norms and informal care provision by women, and that the strength of this link varies within European countries and is strongest for Germany and Southern European countries. Our model explains how these documented facts affect families' LTC arrangements, shows that they reflects an inefficient equilibrium, and studies potentially welfare improving policies.

We consider long-term care choices by daughters and sons in a society in which families consist either of mixed-gender or same-gender siblings. LTC care can be provided 
informally at home, or formally at home or in an institution. LTC decision are made through a cooperative bargaining procedure within each family. Since cooperation guarantees that the outcome is on the family's Pareto frontier, the solution can be characterized by maximizing a weighted sum of parent's and children's utilities. Providing informal care has two types of costs for siblings. The first is the opportunity cost of lost labor income and is gender-specific because of a gender wage gap. The second one is family-specific and captures all the other costs of informal LTC, including material and psychological costs. The first cost is mainly relevant for the intensive margin of informal LTC, while the second one is relevant for the extensive margin (the choice between home care and an institution). A social norm inflicts a psychological cost on daughters when they do not provide enough informal LTC at home. ${ }^{2}$ The cost of the social norm is increasing in the amount of informal LTC provided by the average child in society. The result is that informal care provided by sons and daughters exerts a negative externality on daughters not providing informal care.

We also determine the socially optimal solution, that is the allocation that maximizes a utilitarian social welfare function. Following the tradition of public and welfare economics, social welfare is based on individual (as opposed to family) utility. This introduces a "paternalistic" dimension into social preferences in the sense that society weights individuals equally irrespective of their bargaining weights within their respective families.

We show that the laissez-faire (LF) and the utilitarian first-best (FB) differ for two reasons. First, because informal care imposes a negative externality on daughters via the social norm, the equilibrium will be inefficient. In other words, while the solution is on the Pareto frontier within every family, it is not Pareto efficient for the economy as a whole. The externality will imply too much informal care so that the levels of both

\footnotetext{
${ }^{2}$ Our results do not change when there is also a social norm for sons, as long as their norm cost is lower or not too much larger than the cost for daughters.
} 
formal home and institutional care will be too small. Consequently, it is possible to make both children and parents better off through a suitably designed LTC policy.

Second, the LF differs from the social optimum because the weights children and parents have in the family bargaining problem differ in general from their weights in social welfare. Thus, even when there is no social norm, so that the LF is on the Paretofrontier, the solution will not be socially optimal. In that case a Pareto-improvement is not possible, but the utilitarian social welfare function calls for a move along the Pareto-frontier to make either the caregivers or the parents better off, depending on the family bargaining weights. In the general case where both social norms and different weights apply, the two effects just described are of course intertwined. However, it is insightful to keep them in mind and to look at special cases where only one of them applies.

To study policy design we assume away any ad hoc restrictions on the set of feasible instruments. In particular, subsidies or taxes can be gender-specific. We show that the FB can be implemented through a system of subsidies on formal home and institutional care, financed by a uniform lump-sum tax. When parents and children have identical bargaining weights, so that families maximize the sum of parent's and children's utilities, a simple uniform Pigouvian subsidy is sufficient. The same subsidy rate applies to all modes of formal care (home and institutional) irrespective of the gender of the siblings.

When intra-family weights differ across generations, optimal subsidies must be genderspecific. Both efficiency and paternalistic (weight-related) effects are now relevant. They reinforce each other when parents have the higher weights, so that all the net subsidies are positive and exceed the Pigouvian levels. Daughters are now subject to a double jeopardy and suffer both from the social norm and from their lower weight in the family. Sons, on the other hand, do not care about the social norm, but their informal care contributes to the externality (which is not accounted for in their private decision). In addition, their informal care is inflated (compared to the utilitarian benchmark) by the 
higher weight of the parents. To sum up, too much informal care is provided in the LF and this calls for subsidies on all modes of formal care.

Finally, when children have a higher weight than their parents efficiency and paternalism go in opposite directions. Then, it may become optimal to discourage one or both types of formal care.

\section{The setup}

We consider a population of families. Each family consists of two adult siblings and one old and impaired parent. ${ }^{3}$ Children are randomly matched two by two. Given that $50 \%$ of children are sons and 50\% are daughters, three types of families exist: 1/2 of siblings are mixed, that is they are composed of one son and one daughter $(s, d)$, and $1 / 4$ of siblings are composed of two sons $(s, s)$, or two daughters $(d, d)$ respectively. While parents are economically inactive and only consume care, the children participate in the labor market. The extent to which they do depends on their informal care provision. We normalize all available time to one, and we assume that parents need full-time care.

Two modes of care for the elderly exist: informal LTC provided by adult children and formal market care. Formal care can either be provided at the parent's home, or in a nursing home. We denote informal LTC provision by $a_{i}(i=s, d)$, while that bought in the private market by $a_{p}$. The latter costs $p$ per unit of time. The parent's utility of informal care is given by $u\left(a_{i}+a_{j}\right)(i, j=s, d)$ and it is given by $\beta u\left(a_{p}\right)$ for formal care with $\beta \in(0,1]$. We let $u^{\prime}>0, u^{\prime \prime}<0$ and $u(0)=0$. Informal and private LTC are thus imperfect substitutes, with private LTC being (weakly) less welfare enhancing than informal care. ${ }^{4}$ When old parent enter the nursing home they receive full-time assistance and $a_{p}=1$, with $\beta u(1)>p$.

\footnotetext{
${ }^{3}$ This parent is usually the person who took care of his/her spouse before widowhood.

${ }^{4}$ There are several papers that suggest that different forms of care are, at least to some extent, substitutable (see, e.g., van Houtven and Norton, 2004; Charles and Sevak, 2005; and Stabile et al., 2006).
} 
When adult children provide LTC their labor income decreases proportionally to the time devoted to care. Sons receive an income $w\left(1-a_{s}\right)$ while daughters receive an income $\alpha w\left(1-a_{d}\right)$, where $\alpha \in(0,1]$ reflects the gender wage gap for female workers as it is observed in nearly all developed countries. ${ }^{5}$ A decrease in labor income is not the unique opportunity cost of providing informal LTC. Informal care also comes with material and psychological costs for the care provider and these costs differ between families due to different living situations, or family ties. ${ }^{6}$ We denote those costs $c\left(a_{i}\right)$ and assume that, for a given family, they only take two values: $c(0)=0$ and $c\left(a_{i}\right)=c, \forall a_{i}>0$, where $c \in\left[0, c_{\max }\right]$ is family-specific and is distributed according to the cumulative density function $F(c)$ which is equal for all three types of families.

Daughters are confronted with a social norm concerning LTC activities. This norm is motivated by the empirical evidence mentioned in the introduction showing that women are the principal caregivers in the family. We assume that daughters feel guilt when they provide less informal LTC than the average amount provided by adult children in the society denoted by $\bar{a}$. Formally, we represent the norm for daughters as the costs $\gamma \max \left\{0 ; \bar{a}-a_{d}\right\}$. The parameter $\gamma \in[0,1]$ reflects the psychological costs from norm deviations.

Families maximize the weighted sum of utilities, with $\delta \in(0,1)$ being the weight of the siblings' utility and $1-\delta$ being the weight of the parent's utility. The weights are the same in all families. We define $\Delta \equiv(1-\delta) / \delta$ as the relative weight of the parent. When $\Delta=1 \Leftrightarrow \delta=1 / 2$ the young and old generation have equal weights. When instead $\Delta<(>) 1 \Leftrightarrow \delta>(<) 1 / 2$, the old generation has a lower (higher) weight than the young one. Unequal weights capture situations in which the bargaining power of the two generations differ. To the best of our knowledge no empirical evidence on the relative size of intergenerational weights exists so that both cases are equally possible

\footnotetext{
${ }^{5}$ See, among others, O'Neill (2003); Fortin (2005); Blau and Kahn (2006).

${ }^{6}$ For a more in-depth analysis of the costs of informal care provision see, for instance, Carmichael and Charles (1998).
} 
and the likelihood that one or the other realizes is related to the strength of family ties in society.

To sum up, families differ in two respects: first, in the gender mix of the siblings and, second, in the material and psychological costs $c$.

\section{Families' choices}

In this section we study families' choice of the mode of formal LTC and the siblings' allocation of time between work and informal care in case the old parent remains at home.

Mixed-gender families. First, let us consider a family composed of a son and a daughter. A type- $(s, d)$ family's optimization problem when informal LTC is provided within the family is given by:

$$
\begin{aligned}
\max _{a_{s}, a_{d}} W_{s d}^{1}= & \delta\left[w\left(1-a_{s}\right)+\alpha w\left(1-a_{d}\right)-p a_{p}-c\left(a_{s}\right)-c\left(a_{d}\right)-\gamma \max \left\{0 ; \bar{a}-a_{d}\right\}\right] \\
& +(1-\delta)\left[u\left(a_{s}+a_{d}\right)+\beta u\left(a_{p}\right)\right], \quad \text { s.t. } \quad a_{s}+a_{d}+a_{p}=1 .
\end{aligned}
$$

Given the time constraint, the amount of home formal care is $a_{p}=1-a_{s}-a_{d}$ and the corresponding family's expenditure amount to $p\left(1-a_{s}-a_{d}\right)$.

Together with the decrease in labor income, informal care providers incur a utility cost $c\left(a_{i}\right), i=s, d$. In addition, the daughter is negatively affected by the social norm whenever she provides less informal care than the average amount of care, $\bar{a}$, provided in society.

The first order conditions (FOCs) with respect to the son's and the daughter's informal care provision, $a_{s}$ and $a_{d}$, are given by:

$$
\begin{array}{ll}
a_{s}^{*}: & -\delta[w-p]+(1-\delta)\left[u^{\prime}\left(a_{s}^{*}+a_{d}^{*}\right)-\beta u^{\prime}\left(1-a_{s}^{*}-a_{d}^{*}\right)\right] \leq 0, \\
a_{d}^{*}: \quad & -\delta[\alpha w-p-\gamma I]+(1-\delta)\left[u^{\prime}\left(a_{s}^{*}+a_{d}^{*}\right)-\beta u^{\prime}\left(1-a_{s}^{*}-a_{d}^{*}\right)\right] \leq 0,
\end{array}
$$


where $I \in\{0,1\}$ is an indicator function that takes on the value one when the social norm is binding and zero otherwise. The net marginal costs for each sibling (weighted by the weight of her/his generation) are given by the first term in brackets while the net marginal benefit to the parent (weighted by the weight of her/his generation) is presented by the second term in brackets. Due to the gender wage gap, $\alpha w<w$, and the (possible) costs of the social norm, $\gamma I$, the marginal costs of informal care provision are lower for the daughter than for the son so that only the daughter will provide informal LTC and $a_{s}^{*}=0$. The optimal amount of informal care, $a_{d}^{*}$, is thus implicitly determined by:

$$
a_{d}^{*}: \quad \alpha w-p-\gamma I=\Delta\left[u^{\prime}\left(a_{d}^{*}\right)-\beta u^{\prime}\left(1-a_{d}^{*}\right)\right] .
$$

When the old parent has the lower weight in the family $(\Delta<1)$, the net marginal benefit from informal care is reduced and $a_{d}^{*}$ decreases accordingly.

When informal LTC is provided, welfare in the $(s, d)$ family is given by: $W_{s d}^{1 *}=\delta\left[w+\alpha w\left(1-a_{d}^{*}\right)-p\left(1-a_{d}^{*}\right)-c-\gamma \max \left\{0 ; \bar{a}-a_{d}^{*}\right\}\right]+(1-\delta)\left[u\left(a_{d}^{*}\right)+\beta u\left(1-a_{d}^{*}\right)\right]$.

The alternative to informal LTC provision is a nursing home. In this case $a_{p}^{*}=1$ and the disutility from the social norm suffered by the daughter is $\gamma \max \{0 ; \bar{a}-0\}=\gamma \bar{a}$. Welfare of a type- $(s, d)$ family is then:

$$
W_{s d}^{0 *}=\delta[w+\alpha w-p-\gamma \bar{a}]+(1-\delta) \beta u(1) .
$$

The family opts for the nursing home if it is welfare maximizing, that is if

$$
W_{s d}^{1 *} \leq W_{s d}^{0 *} \quad \Leftrightarrow \quad \hat{c}_{s d}^{*} \leq c
$$

where the critical costs, $c_{s d}^{*}$, are defined by:

$$
\hat{c}_{s d}^{*} \equiv\left[(p-\alpha w) a_{d}^{*}+\gamma \max \left\{0 ; \bar{a}-a_{d}^{*}\right\}+\gamma \bar{a}\right]+\Delta\left[u\left(a_{d}^{*}\right)+\beta\left[u\left(1-a_{d}^{*}\right)-u(1)\right]\right] .
$$

For the share $F\left(\hat{c}_{s d}^{*}\right)$ of type- $(s, d)$ families it is welfare maximizing to provide informal care while for the share $1-F\left(\hat{c}_{s d}^{*}\right)$ of these families it is optimal that the parent enters 
a nursing home. The critical cost level and thus the share of families providing informal care is thereby increasing in the norm $\operatorname{costs} \gamma$, and in the relative weight of the parent, $\Delta$. Hence, when $\Delta<1$, the two economic forces push in opposite directions: the social norm causes the share of families choosing a nursing home to decrease and a lower weight for the parent causes it to increase. ${ }^{7}$

Two-daughter families. Now consider a family with two daughters $(d, d)$. When they provide informal care, their optimization problem is given by:

$$
\begin{aligned}
\max _{a_{d 1}, a_{d 2}} W_{d d}^{1}= & \delta\left[\alpha w\left(1-a_{d 1}\right)+\alpha w\left(1-a_{d 2}\right)-p a_{p}-c\left(a_{d 1}\right)-c\left(a_{d 2}\right)\right. \\
& \left.-\gamma\left\{0 ; \bar{a}-a_{d 1}\right\}-\gamma\left\{0 ; \bar{a}-a_{d 2}\right\}\right]+(1-\delta)\left[u\left(a_{d 1}+a_{d 2}\right)+\beta u\left(a_{p}\right)\right] .
\end{aligned}
$$

Differently from before, both daughters are possibly negatively affected by the social norm. Noting that $a_{p}=1-a_{d 1}-a_{d 2}$, the FOC with respect to both $a_{d 1}$ and $a_{d 2}$ is given by:

$$
a_{d 1}^{*}, a_{d 2}^{*}: \quad-\delta[\alpha w-p-\gamma I]+(1-\delta)\left[u^{\prime}\left(a_{d 1}^{*}+a_{d 2}^{*}\right)-\beta u^{\prime}\left(1-a_{d 1}^{*}-a_{d 2}^{*}\right)\right] \leq 0 .
$$

Since the FOCs of the two daughters coincide, only one of them will provide informal LTC so that the fixed cost $c$ is not duplicated. We assume this to be daughter $d 1$ so that $a_{d 2}^{*}=0$ and $a_{d 1}^{*}$ is implicitly determined by:

$$
a_{d 1}^{*}: \quad \alpha w-p-\gamma I=\Delta\left[u^{\prime}\left(a_{d 1}^{*}\right)-\beta u^{\prime}\left(1-a_{d 1}^{*}\right)\right] .
$$

Note that informal LTC in a two-daughter family coincides with informal care in a mixed family (see expression 1 ) and we define $a_{d 1}^{*} \equiv a_{d}^{*}$. The daughter who is not providing

\footnotetext{
${ }^{7}$ Accounting for a social norm for sons would imply a cost $\gamma_{s} \max \left\{0 ; \bar{a}-a_{s}\right\}$. Results would not change qualitatively provided that the cost of deviating from the norm is not more salient for sons $\left(\gamma_{s}\right.$ should not be too high). The results would be reversed with sons providing care in mixed gender families only when $\gamma_{s}>\gamma_{d}$, and the difference is sufficiently large to outweigh the difference in opportunity costs implied by the gender wage gap.
} 
informal care is suffering a disutility from the social norm given by $\gamma \bar{a}$. Welfare in a type- $(d, d)$ family with informal care provision can thus be written as:

$$
\begin{aligned}
W_{d d}^{1 *}= & \delta\left[\alpha w\left(1-a_{d}^{*}\right)+\alpha w-p\left(1-a_{d}^{*}\right)-c-\gamma \max \left\{0 ; \bar{a}-a_{d}^{*}\right\}-\gamma \bar{a}\right] \\
& +(1-\delta)\left[u\left(a_{d}^{*}\right)+\beta u\left(1-a_{d}^{*}\right)\right] .
\end{aligned}
$$

If a type- $(d, d)$ family instead fully relies on market care, welfare amounts to:

$$
W_{d d}^{0 *}=\delta[2 \alpha w-p-2 \gamma \bar{a}]+(1-\delta) \beta u(1)
$$

Now the cost of the social norm is counted twice, because both sisters feel guilt when their parent enters the nursing home. The latter will be the case if it yields a higher welfare, that is if

$$
W_{d d}^{1 *} \leq W_{d d}^{0 *} \quad \Leftrightarrow \quad \hat{c}_{d d}^{*} \leq c
$$

where

$$
\hat{c}_{d d}^{*} \equiv\left[(p-\alpha w) a_{d}^{*}+\gamma \max \left\{0 ; \bar{a}-a_{d}^{*}\right\}+\gamma \bar{a}\right]+\Delta\left[u\left(a_{d}^{*}\right)+\beta\left[u\left(1-a_{d}^{*}\right)-u(1)\right]\right] .
$$

Given that all daughters provide the same amount of informal care $a_{d}^{*}$, the critical values $\hat{c}_{d d}^{*}=\hat{c}_{s d}^{*} \equiv \hat{c}_{d}^{*}$ are the same, as well as the share of $(d, d)$ and $(s, d)$ families for whom it is better to provide informal care: $F\left(\hat{c}_{d d}^{*}\right)=F\left(\hat{c}_{s d}^{*}\right) \equiv F\left(\hat{c}_{d}^{*}\right)$.

Two-son families. Finally, consider a family with two sons $(s, s)$. The social norm is not relevant in this family and the optimization problem writes:

$$
\begin{aligned}
& \max _{a_{s 1}, a_{s 2}} W_{s s}^{1}= \delta\left[w\left(1-a_{s 1}\right)+w\left(1-a_{s 2}\right)-p a_{p}-c\left(a_{s 1}\right)-c\left(a_{s 2}\right)\right] \\
&+(1-\delta)\left[u\left(a_{s 1}+a_{s 2}\right)+\beta u\left(1-a_{p}\right)\right]
\end{aligned}
$$

Noting that $a_{p}=1-a_{s 1}-a_{s 2}$ the FOCs with respect to both $a_{s 1}$ and $a_{s 2}$ is given by:

$$
a_{s 1}^{*}, a_{s 2}^{*}: \quad-\delta[w-p]+(1-\delta)\left[u^{\prime}\left(a_{s 1}^{*}+a_{s 2}^{*}\right)-\beta u^{\prime}\left(1-a_{s 1}^{*}-a_{s 2}^{*}\right)\right] \leq 0
$$


Again, to avoid cost duplication only one son will provide care, which we assume to be son $s 1$ so that $a_{s 2}^{*}=0$. Hence, informal LTC in a two-son family $a_{s}^{*} \equiv a_{s 1}^{*}$ is chosen such that the net opportunity costs in terms of labor income equals the (weighted) net marginal benefit of informal LTC to the parent

$$
a_{s}^{*}: \quad w-p=\Delta\left[u^{\prime}\left(a_{s}^{*}\right)-\beta u^{\prime}\left(1-a_{s}^{*}\right)\right] .
$$

Comparing (1) with (2) reveals that $a_{s}^{*}<a_{d}^{*}$ since both the gender wage gap and the norm costs reduce the opportunity cost of informal LTC provision for daughters. In a type- $(s, s)$ family welfare when informal care is provided can thus be written as:

$$
W_{s s}^{1 *}=\delta\left[w\left(1-a_{s}^{*}\right)+w-p\left(1-a_{s}^{*}\right)-c\right]+(1-\delta)\left[u\left(a_{s}^{*}\right)+\beta u\left(1-a_{s}^{*}\right)\right] .
$$

When instead the parent enters the nursing home, welfare is given by:

$$
W_{s s}^{0 *}=\delta[2 w-p]+(1-\delta) \beta u(1)
$$

The nursing home is welfare maximizing if

$$
W_{s s}^{1 *} \leq W_{s s}^{0 *} \quad \Leftrightarrow \quad \hat{c}_{s s}^{*} \leq c
$$

where

$$
\hat{c}_{s s}^{*} \equiv(p-w) a_{s}^{*}+\Delta\left[u\left(a_{s}^{*}\right)+\beta\left[u\left(1-a_{s}^{*}\right)-u(1)\right]\right] .
$$

For type- $(s, s)$ families the social norm does not affect the critical cost level $\hat{c}_{s s}^{*}$ and thus the share of families providing informal care $F\left(\hat{c}_{s s}^{*}\right)$; however, when the parent has the lower weight in the family $(\Delta<1)$ this still reduces $F\left(\hat{c}_{s s}^{*}\right){ }^{8}$

\section{Laissez-faire allocation}

We are now in the position to characterize the laissez faire allocation. To do so, we first derive the average amount of informal care provided in the population. Then, we show how the social norm affects daughters in our society.

\footnotetext{
${ }^{8}$ The model can account for only-child families. Our ananysis immediatly implies that the unique child would behave either as sibling $d_{1}$ or as sibling $s_{1}$ of same-gender families.
} 
Recall that daughters provide the same level of informal care, $a_{d}^{*}$, in type- $(s, d)$ and $(d, d)$ families. The latter correspond to $1 / 2$ and $1 / 4$ of families in the population. Moreover, the critical cost value, $\hat{c}_{d}^{*}$, is the same in both types of families. Average informal care in the society is thus given by:

$$
\begin{aligned}
\bar{a}^{*} & =\left(\frac{1}{2} F\left(\hat{c}_{s d}^{*}\right)+\frac{1}{4} F\left(\hat{c}_{d d}^{*}\right)\right) a_{d}^{*}+\frac{1}{4} F\left(\hat{c}_{s s}^{*}\right) a_{s}^{*} \\
& =\frac{3}{4} F\left(\hat{c}_{d}^{*}\right) a_{d}^{*}+\frac{1}{4} F\left(\hat{c}_{s s}^{*}\right) a_{s}^{*} .
\end{aligned}
$$

where $a_{d}^{*}$ and $a_{s}^{*}$ are defined in (1) and (2) respectively. Given that $a_{s}^{*}<a_{d}^{*}$, expression (3) shows that $a_{s}^{*}<\bar{a}<a_{d}^{*}$, or that daughters provide more than the average amount of informal care while sons provide less than the average amount of care in society. The latter implies that the norm is never binding for daughters who provide informal care so that $I=0$ and

$$
a_{d}^{*}: \quad \alpha w-p=\Delta\left[u^{\prime}\left(a_{d}^{*}\right)-\beta u^{\prime}\left(1-a_{d}^{*}\right)\right]
$$

Let us sum up the impact of the social norm on the laissez-faire allocation. In families in which informal care is provided, the norm uniquely affects type- $(d, d)$ families where the sister who is working full-time feels guilt of not providing care. However, the social norm has always an impact on families with at least one daughter if the parent enters the nursing home.

The critical cost level within the three family types can thus be rewritten as:

$$
\begin{aligned}
\hat{c}_{s d}^{*}=\hat{c}_{d d}^{*} & =\hat{c}_{d}^{*} \equiv(p-\alpha w) a_{d}^{*}+\gamma \bar{a}^{*}+\Delta\left[u\left(a_{d}^{*}\right)+\beta\left[u\left(1-a_{d}^{*}\right)-u(1)\right]\right] \\
\hat{c}_{s s}^{*} & =\hat{c}_{s}^{*} \equiv(p-w) a_{s}^{*}+\Delta\left[u\left(a_{s}^{*}\right)+\beta\left[u\left(1-a_{s}^{*}\right)-u(1)\right]\right] .
\end{aligned}
$$

It is larger in type- $(s, d)$ and $(d, d)$ families than in type- $(s, s)$ families: $\hat{c}_{s}^{*}<\hat{c}_{d}^{*}$. This translates in a larger share of type- $(s, s)$ families where the parent enters the nursing home, that is $F\left(\hat{c}_{s}^{*}\right)<F\left(\hat{c}_{d}^{*}\right)$.

The following proposition provides a characterization of the laissez-faire allocation. 
Proposition 1 (Laissez-faire allocation) (i) When the parent does not enter the nursing home, informal LTC is provided by a single sibling. Except in type- $(s, s)$ families this will be a daughter.

(ii) The levels of informal care are determined by (2) and (4). They satisfy: $a_{s}^{*}<$ $\bar{a}^{*}<a_{d}^{*}$, where $\bar{a}^{*}$ is the average level of informal care defined by (3).

(iii) Daughters characterized by a cost c lower than $\hat{c}_{d}^{*}$, in type- $(s, d)$ and $(d, d)$ families, will provide informal care. Sons characterized by a cost c lower that $\hat{c}_{s}^{*}$ will provide informal care in type- $(s, s)$ families. The marginal siblings $\hat{c}_{d}^{*}$ and $\hat{c}_{s}^{*}$ are defined by (5) and (6) respectively, and are such that $\hat{c}_{s}^{*}<\hat{c}_{d}^{*}$.

(iv) All daughters not providing informal care suffer from the presence of the social norm.

The cost of the social norm depends on $\bar{a}^{*}$, families' decisions are thus related in many ways. First, in all type- $(d, d)$ and $(s, d)$ families opting for the nursing home, the disutility from the social norm is affected by the choices $a_{s}^{*}$ and $a_{d}^{*}$ taken by all families providing informal care. Second, the cost value of the indifferent sibling $\hat{c}_{d}^{*}$ not only depends on the amount of informal care provided by daughters in the corresponding type of family, but also on informal care decisions made by all other types of families and on the indifferent siblings in type- $(s, s)$ families, $\hat{c}_{s}^{*}$ (see expression 5 ).

\section{First-best allocation}

We now characterize the first-best solution in order to analyze the laissez-faire inefficiencies created by the existence of the social norm on the one side, and by the unequal generational weights on the other side. We consider a utilitarian social welfare function given by the sum of utilities of the three types of families, weighted by their respective 
shares, and which takes the social norm into account. In other words, welfare corresponds to the sum of utility of all individuals. Observe that this is in line with families' objective function when $\Delta=1$, which corresponds precisely to the case where the parent receives the same weight as each of the children. ${ }^{9}$ When $\Delta \neq 1$, social and family weights differ which introduces a "paternalistic" dimension.

Recall that an allocation specifies the amount of informal LTC provided by sons and daughters, and the individual cost of providing informal LTC characterizing the siblings who are indifferent between providing and not providing informal LTC in the three types of families. Hence, we have to determine $a_{s s}, a_{s d}, a_{d d}, \hat{c}_{s s}, \hat{c}_{s d}$ and $\hat{c}_{d d}$ that maximize the following social welfare function:

$$
\begin{aligned}
\max _{a_{s s}, a_{s d}, a_{d d}, \hat{c}_{s s}, \hat{c}_{s d}, \hat{c}_{d d}} S W & =\frac{1}{2}\left\{\int_{0}^{\hat{c}_{s d}} W_{s d}^{1} f(c) \mathrm{d} c+\left[1-F\left(\hat{c}_{s d}\right)\right] W_{s d}^{0}\right\} \\
& +\frac{1}{4}\left\{\int_{0}^{\hat{c}_{d d}} W_{d d}^{1} f(c) \mathrm{d} c+\left[1-F\left(\hat{c}_{d d}\right)\right] W_{d d}^{0}\right\} \\
& +\frac{1}{4}\left\{\int_{0}^{\hat{c}_{s s}} W_{s s}^{1} f(c) \mathrm{d} c+\left[1-F\left(\hat{c}_{s s}\right)\right] W_{s s}^{0}\right\},
\end{aligned}
$$

where, given that the young and old generation are weighted equally, families welfare

\footnotetext{
${ }^{9}$ In the original specification with $\delta$, each family maximizes (with obvious notation) $\delta U_{P}+(1-$ $\delta)\left(U_{C 1}+U_{C 2}\right)$. With $\delta=1 / 2$ (or $\Delta=1$ ) this yields

$$
\frac{1}{2}\left(U_{P}+U_{C 1}+U_{C 2}\right)
$$

which corresponds to the maximization of the sum of utilities. We obtain the same social welfare function (7) if each individual is given a weight of $1 / 3$. All this is just a matter of normalization.
} 
levels are given by:

$$
\begin{aligned}
& W_{s d}^{1}=w+\alpha w\left(1-a_{d}\right)-p\left(1-a_{d}\right)-c-\gamma \max \left\{0 ; \bar{a}-a_{d}\right\}+u\left(a_{d}\right)+\beta u\left(1-a_{d}\right), \\
& W_{s d}^{0}=w+\alpha w-p-\gamma \bar{a}+\beta u(1), \\
& W_{d d}^{1}=\alpha w\left(1-a_{d 1}\right)+\alpha w-p\left(1-a_{d 1}\right)-c-\gamma \max \left\{0 ; \bar{a}-a_{d 1}\right\}-\gamma \bar{a}+u\left(a_{d 1}\right)+\beta u\left(1-a_{d 1}\right), \\
& W_{d d}^{0}=2 \alpha w-p-2 \gamma \bar{a}+\beta u(1), \\
& W_{s s}^{1}=w\left(1-a_{s 1}\right)+w-p\left(1-a_{s 1}\right)-c+u\left(a_{s 1}\right)+\beta u\left(1-a_{s 1}\right), \\
& W_{s s}^{0}=2 w-p+\beta u(1) .
\end{aligned}
$$

Note that, in the above expressions for families' welfare $\left(W_{s d}^{1}, W_{d d}^{1}\right.$ and $\left.W_{s s}^{1}\right)$ only one sibling is providing informal care in each family so that fixed costs are not duplicated. Moreover, in type- $(s, d)$ families it is optimal, given the gender wage gap, that the daughter takes the role of the caregiver.

The optimal levels of informal LTC are derived in Appendix A. As in the laissez faire, daughters in type- $(s, d)$ and in $(d, d)$ families provide the same amount of informal care $\left(a_{s d}^{F B}=a_{d d}^{F B} \equiv a_{d}^{F B}\right)$ which solves:

$$
a_{d}^{F B}: \quad \alpha w-p+\varphi=u^{\prime}\left(a_{d}^{F B}\right)-\beta u^{\prime}\left(1-a_{d}^{F B}\right) .
$$

Informal care provided by sons in type- $(s, s)$ families instead solves:

$$
a_{s}^{F B}: \quad w-p+\varphi=u^{\prime}\left(a_{s}^{F B}\right)-\beta u^{\prime}\left(1-a_{s}^{F B}\right) .
$$

The right hand side (RHS) in (8) and (9) reflects the net marginal benefit of informal care provision to the parent while the left hand side (LHS) indicates the sum of the private and the social marginal cost of informal care. In particular, the social marginal cost of informal care is captured by the term $\varphi$ and is defined by:

$$
\varphi \equiv \gamma\left(\frac{1}{2}\left[1-F\left(\hat{c}_{s d}^{F B}\right)\right]+2 \frac{1}{4}\left[1-F\left(\hat{c}_{d d}^{F B}\right)\right]+\frac{1}{4} F\left(\hat{c}_{d d}^{F B}\right)\right) .
$$


The above term collects all marginal effects of informal care provided by sons and daughters on the average amount of informal care $\bar{a}^{F B}$ and its expression reflects the total negative externality imposed by informal care providers on all daughters who do not provide care. Specifically, the first two terms in brackets reflect the costs of all daughters in type- $(s, d)$ and $(d, d)$ families in which parents enter the nursing home. ${ }^{10}$ The third term captures the costs of sisters not providing informal care in type- $(d, d)$ families where parents stay at home. Note that, due to the gender wage gap, it is optimal that daughters provide (weakly) more informal care than sons, i.e. $a_{s}^{F B} \leq a_{d}^{F B}$.

As shown in Appendix A the efficient critical cost level for daughters $\hat{c}_{s d}^{F B}=\hat{c}_{d d}^{F B} \equiv$ $\hat{c}_{d}^{F B}$ and sons $\hat{c}_{s s}^{F B} \equiv \hat{c}_{s}^{F B}$ are given by:

$$
\begin{aligned}
& \hat{c}_{d}^{F B} \equiv(p-\alpha w) a_{d}^{F B}+\gamma \bar{a}^{F B}+u\left(a_{d}^{F B}\right)+\beta\left[u\left(1-a_{d}^{F B}\right)-u(1)\right]-\varphi a_{d}^{F B}, \\
& \hat{c}_{s}^{F B} \equiv(p-w) a_{s}^{F B}+u\left(a_{s}^{F B}\right)+\beta\left[u\left(1-a_{s}^{F B}\right)-u(1)\right]-\varphi a_{s}^{F B} .
\end{aligned}
$$

As in the laissez-faire, given that all female caregivers optimally provide the same amount of informal care, the indifferent daughter in type- $(s, d)$ and $(d, d)$ families is the same. In addition, it is optimal that the share of type- $(s, s)$ families in which the parent enters the nursing home is larger than the corresponding share of type- $(s, d)$ and $(d, d)$ families:

$$
\hat{c}_{s}^{F B} \leq \hat{c}_{d}^{F B}
$$

Finally, the optimal average informal care in society is given by:

$$
\begin{aligned}
\bar{a}^{F B} & =\left(\frac{1}{2} F\left(\hat{c}_{s d}^{F B}\right)+\frac{1}{4} F\left(\hat{c}_{d d}^{F B}\right)\right) a_{d}^{F B}+\frac{1}{4} F\left(\hat{c}_{s s}^{F B}\right) a_{s}^{F B} \\
& =\frac{3}{4} F\left(\hat{c}_{d}^{F B}\right) a_{d}^{F B}+\frac{1}{4} F\left(\hat{c}_{s}^{F B}\right) a_{s}^{F B}
\end{aligned}
$$

which is composed of the same terms as in the laissez-faire, but where the values of $\hat{c}_{d}, a_{d}, \hat{c}_{s}$ and $a_{s}$ differ, as we will discuss in the next section. Considering that $\hat{c}_{s d}^{F B}=$

\footnotetext{
${ }^{10}$ The second term in (10) is counted twice because there are two sisters in type- $(d, d)$ families.
} 
$\hat{c}_{d d}^{F B} \equiv \hat{c}_{d}^{F B}$, the social marginal costs $\varphi$ defined in (10) simplify to:

$$
\varphi \equiv \gamma\left(1-\frac{3}{4} F\left(\hat{c}_{d}^{F B}\right)\right)
$$

where the costs of deviating from the social norm, $\gamma$, are multiplied by the whole share of daughters suffering from the norm in the society.

The results of this section are summarized in the following proposition.

\section{Proposition 2 (First-best allocation) (i) When the parent does not enter the nurs-} ing home, informal LTC is provided by a single sibling. Except in type- $(s, s)$ families this will always be a daughter.

(ii) The optimal levels of informal care are determined by (8) and (9) and satisfy $a_{s}^{F B}<\bar{a}^{F B}<a_{d}^{F B}$, where $\bar{a}^{F B}$ is the average level of informal care defined by (13). $a_{s}^{F B}$ and $a_{d}^{F B}$ take into account the marginal social cost of informal care, $\varphi$, defined in (14), or the negative externality imposed by informal care providers on all daughters who are not providing informal care.

(iii) Daughters characterized by a cost c lower than $\hat{c}_{d}^{F B}$, in type- $(s, d)$ and $(d, d)$ families, will provide informal care. Sons characterized by a cost $c$ lower than $\hat{c}_{s}^{F B}$ will provide informal care in type- $(s, s)$ families. The marginal siblings $\hat{c}_{d}^{F B}$ and $\hat{c}_{s}^{F B}$ are defined by (11) and (12) and satisfy $\hat{c}_{s}^{F B}<\hat{c}_{d}^{F B}$.

\section{Policy design}

The results presented in the previous sections have shown that the LF and the utilitarian FB differ for two reason. First, through the social norm, informal care imposes a negative externality on daughters. Because of this externality the equilibrium will be inefficient. Ceteris paribus it will imply too much informal care so that the levels of both formal home and institutional care will be too small. Observe that this inefficiency implies that 
the equilibrium is not on the Pareto frontier. Consequently, it is possible to make both children and parents better off through a suitably designed LTC policy.

Second, the LF differs from the social optimum because the weights children and parents have in the family bargaining problem differ in general (unless $\Delta=1$ ) from their weights in social welfare. Thus, even when there is no social norm, so that the LF is on the Pareto-frontier, the solution will not be socially optimal. In that case a Pareto-improvement is not possible but the utilitarian social welfare function calls for a move along the Pareto-frontier to make either the caregivers or the parents better off, depending on the family bargaining weights. In the general case where both social norms and different weights apply, the two effects just described are of course intertwined. However, it is insightful to keep them in mind and to look at special cases where only one of them applies.

We now turn to the design of long term care policies. Specifically, we examine how the FB allocation can be implemented through such policies. The following proposition is established in Appendix B.

Proposition 3 The utilitarian first-best solution can be implemented by a system of linear gender-specific (positive or negative) subsidies on formal home care, $\sigma_{s}$ and $\sigma_{d}$, and on institutional care, $\theta_{s}$ and $\theta_{d}$, financed by a uniform lump-sum tax. The implementing subsidies are given by

$$
\begin{aligned}
\sigma_{s} & =(\Delta-1)[w-p]+\Delta \varphi \\
\sigma_{d} & =(\Delta-1)[\alpha w-p]+\Delta \varphi
\end{aligned}
$$

for formal home care and by

$$
\begin{aligned}
& \theta_{s}=\left(1-a_{s}^{F B}\right) \sigma_{s}+\varphi a_{s}^{F B}+(\Delta-1)\left[u\left(a_{s}^{F B}\right)+\beta\left[u\left(1-a_{s}^{F B}\right)-u(1)\right]\right] \\
& \theta_{d}=\left(1-a_{d}^{F B}\right) \sigma_{d}+\varphi a_{d}^{F B}+(\Delta-1)\left[u\left(a_{d}^{F B}\right)+\beta\left[u\left(1-a_{d}^{F B}\right)-u(1)\right]\right]
\end{aligned}
$$


for institutional care, where $\sigma_{s}$ and $\theta_{s}$ apply when both siblings are male while $\sigma_{d}$ and $\theta_{d}$ apply when at least one of the siblings is female.

The expressions for these subsidies reflect the two effects just mentioned. Furthermore, the signs of the subsidies tell us which of the two effects is stronger and in which direction the LF has to be corrected to achieve the optimal solution. More precisely, using expressions (2) and (4) for the LF, and (8) (9) for the FB, it follows that

$$
\begin{aligned}
\sigma_{s} \gtreqless 0 & \Leftrightarrow \quad a_{s}^{*} \gtreqless a_{s}^{F B}, \\
\sigma_{d} \gtreqless 0 & \Leftrightarrow \quad a_{d}^{*} \gtreqless a_{d}^{F B} .
\end{aligned}
$$

In words, the subsidy on formal home care in any given family is positive if the level of informal care provided in the LF is too large and it is negative in the opposite case. This is quite in line with intuition: when the LF implies too much informal care we can reduce it by subsidizing the closest substitute, namely formal home care.

For institutional care the interpretation of the sign is a bit more complicated. First, to get a meaningful expression to interpret we have to consider the net subsidies, rather than just the value of $\theta_{s}$ and $\theta_{d}$. This is given by $\theta_{s}-\left(1-a_{s}^{F B}\right) \sigma_{s}$ for two-son families and by $\theta_{d}-\left(1-a_{d}^{F B}\right) \sigma_{d}$ for all others. These expressions effectively measure the extra subsidy a family receives when switching from formal home to institutional care and it is of course this level which is relevant for the choice between the two modes of care. Second, the comparisons depend on $a_{s}$ and $a_{d}$ which are not the same in the LF and in the FB. In type- $(s, s)$ families, defining the LF and FB levels of $\hat{c}_{s}$ 's conditional on given levels of $a_{s}$ and $a_{d}$, we obtain from (6) and (12) that

$$
\hat{c}_{s}^{F B}\left(a_{s}\right) \gtreqless \hat{c}_{s}^{*}\left(a_{s}\right) \Leftrightarrow(1-\Delta)\left[u\left(a_{s}\right)+\beta\left[u\left(1-a_{s}\right)-u(1)\right]\right] \gtreqless \varphi a_{s},
$$

so that from (17) we have

$$
\hat{c}_{s}^{F B}\left(a_{s}^{F B}\right) \gtreqless \hat{c}_{s}^{*}\left(a_{s}^{F B}\right) \quad \Leftrightarrow \quad \theta_{s}-\left(1-a_{s}^{F B}\right) \sigma_{s} \lesseqgtr 0 .
$$


Roughly speaking, for the sons the net subsidy on institutional care is positive when the LF level of $\hat{c}$ is too large, so that not enough institutional care is provided. ${ }^{11}$

For the daughters the interpretation of the expression is more complicated, because the expression for the cost levels of the marginal daughters in the LF, (5), and in the FB, (11), are not closed-form solutions, as the $\bar{a}$ on the RHS depends on the $\hat{c}$ 's. We shall nevertheless examine the sign of the net subsidy and take it as an indication in which direction the level of institutional care needs to be corrected, keeping in mind that LF and FB levels of $\hat{c}$ have to be compared conditional on given levels of $a$. Though intuitive and formally correct, our comparison thus has to be interpreted with care.

To understand the trade-offs which are involved, it is interesting to consider the two special cases (equal weights and no norm) already mentioned. When family and social weights coincide, we have

$$
\Delta=1 \quad \Leftrightarrow \quad \theta_{s}=\theta_{d}=\sigma_{s}=\sigma_{d}=\varphi
$$

In that case the solution is particularly simple. Not surprisingly a uniform subsidy on all types of formal care which is set according to the Pigouvian rule equal to the "marginal social damage" is sufficient. The uniformity of the subsidy is due to the fact that a children's informal care affects $\bar{a}$ in the same way, irrespective of their gender. In other words, while the norm affects only daughters, the benchmark (average) level of care is gender neutral.

In the other extreme case, when there is no social norm we have $\gamma=\varphi=0$. Expressions (15) and (18) then imply that the subsidies on formal home care $\sigma_{s}$ and $\sigma_{d}$ as well as the net subsidy on institutional care, $\theta_{s}-\left(1-a_{s}^{F B}\right) \sigma_{s}$ and $\theta_{d}-\left(1-a_{d}^{F B}\right) \sigma_{d}$ have the same sign as $(\Delta-1) .{ }^{12}$ The policy is now solely determined by "paternalistic" considerations. When parents have the larger bargaining weight in the family, too much informal care is provided and both modes of formal care, home and institutional, have

\footnotetext{
${ }^{11}$ This comparison is made when formal home care is at its first-best level.

${ }^{12}$ As long as $\alpha w-p>0$ which we assume.
} 
to be subsidized. The opposite result obtains when children have the larger weight. No policy intervention is needed when $\Delta=1$.

In the general case, when $\Delta \neq 1$ and $\gamma>0$, both the efficiency and paternalistic (weight-related) effects are relevant. They reinforce each other when $\Delta>1$ so that all the net subsidies are positive and exceed the Pigouvian levels. Daughters are now subject to a double jeopardy and suffer both from the social norm and from their lower weight in the family. Sons, on the other hand, do not care about the social norm but their informal care contributes to the externality (which is not accounted for in their private decision). In addition, their informal care is inflated (compared to the utilitarian benchmark) by the higher weight of the parents. To sum up, too much informal care is provided in the LF and this calls for subsidies on all modes of formal care.

Finally, when $\Delta<1$ efficiency and paternalism go in opposite directions. By continuity all net subsidies will remain positive when $\Delta$ is sufficiently close to 1 , but the signs may be reversed when $\Delta$ is sufficiently small.

Note that except when $\Delta=1$, subsidies must be gender-specific. Because of the gender wage gap we have $\sigma_{s}>\sigma_{d}$ when $\Delta>1$ while $\Delta<1$ yields the opposite result, namely $\sigma_{s}<\sigma_{d}$. In this case formal home care may have to be subsidized in families with at least one daughter, while it is taxed in two-son families; see expressions (15) and (18). This occurs when

$$
\frac{1-\Delta}{\Delta}(\alpha w-p)<\varphi<\frac{1-\Delta}{\Delta}(w-p) .
$$

The comparison across genders of the net subsidies on institutional care, on the other hand, appears to be ambiguous.

The main properties of the optimal subsidies on formal home and institutional care are summarized in the following proposition.

Proposition 4 The optimal subsidies on informal care stated in Proposition 3 have the following properties: 
(i) When $\Delta=1$, so that family and social weights coincide, we have $\theta_{s}=\theta_{d}=\sigma_{s}=$ $\sigma_{d}=\varphi$ so that a uniform subsidy on all types of formal care which is set according to the Pigouvian rule equal to the "marginal social damage" is sufficient.

(ii) When $\gamma=\varphi=0$ so that there is no social norm the subsidies on formal home care and the net subsidies on institutional care, have the same sign as $(\Delta-1)$. The policy is then gender-specific and solely determined by "paternalistic" considerations.

(iii) In the general case, when $\Delta \neq 1$ and $\gamma>0$, both the efficiency and paternalistic (weight-related) effects are relevant.

- When $\Delta>1$, they reinforce each other so that all the net gender-specific subsidies are positive and larger than the Pigouvian levels.

- When $\Delta<1$ efficiency and paternalism go in opposite directions and the (net) gender-specific subsidies may even become negative.

\section{Concluding comments}

Dependency and the need for LTC are not new problems. However, until rather recently, these topics have received little attention, both in the public debate as in the economic literature. As explained by Cremer et al. (2012), much of this literature is empirical, but there has been an increasing number of theoretical papers over the last few years. Most of these papers look at the problem of LTC from the parent's perspective and they generally neglect welfare of the caregivers. ${ }^{13}$ More importantly, they remain agnostic about caregivers' gender, despite the fact that daughters typically pay the larger burden of informal care. Since "each unhappy family is unhappy in its own way" the type of

\footnotetext{
${ }^{13}$ Konrad et al. (2002) consider parents and children's decisions but concentrate on locational decisions. Klimaviciute (2015) and Canta and Cremer (2017) take the welfare of caregivers into account but continue to study the decisions from the parent's perspective.
} 
interaction between generations is likely to differ across families and altruistic, strategic, exchange or norm based patterns can be expected to coexist in practice. Since an allencompassing model is still out of reach the approach so far has consisted in looking at the various scenarios in isolation.

We continue with this tradition even though we take an otherwise completely unusual and fresh look at the issue of LTC. In particular, we view the provision of LTC as a matter of family bargaining in which caregivers and their gender specific roles are a crucial ingredients. In our setting we abstract from a number of issues which are important and have been stressed so far in the literature (these include risk, insurance, misperception and redistribution) to focus instead on the tradeoff between the caregivers' welfare and their parents needs (or preferences) for informal care. While crowding out of informal care by social (or private) insurance represents a major concern in the existing literature, our paper shows that this view can be misleading. When daughters feel compelled to provide informal care, even in a globally cooperative setting, there may well be too much informal care and public policy ought to subsidize formal care, both at home and in institutions even when issues of redistribution, risk or insurance are neglected. While the existing literature has shown that various policies may be desirable in spite of the crowding out, we argue that policies may be designed to deliberately discourage informal care.

While we deal with gender-specific informal care, it is important to keep in mind that the paper is about inefficiency and not gender equality per se. And while our policies can mitigate the inefficiencies brought about by these inequalities and particularly by both the social norm and the gender wage gap, they have no leverage on the source of these inequalities. The social norm is taken as given and, in our setting, there is nothing that can be done about it. The natural next step would be to make it endogenous, for instance by making it dependent on the behavior of previous generations like in Barigozzi et al. (2017). More fundamentally still is the gender wage gap. As long as 
it is present, daughters will be ceteris paribus natural candidates to provide informal care. While subsidizing formal care may provide a patch, only labor market policies that address the gender wage gap can provide a cure. These are not included in our model, but our analysis shows that they appear to be the only final solution to the problem that daughters provide an "excessive" amount of informal care.

\section{References}

[1] Arber S. and J. Ginn (1990), "The meaning of informal care: gender and the contribution of elderly people", Ageing and Society, 10 (4), 429-454.

[2] Barigozzi, F., H. Cremer and K. Roeder (2017), "Women's career choices, social norms and child care policies", TSE WP 17-754, 2017.

[3] Blau, F.D., and L.M. Kahn, "The US gender pay gap in the 1990s: Slowing convergence," Industrial and Labor Relations Review, 2006, 60 (1), 45-66.

[4] Bott, N.T., C.C. Sheckter, A.S. Milstein (2017), "Dementia care, women's health, and gender equity: the value of well-timed caregiver support", JAMA Neurol. Published online May 08, doi:10.1001/jamaneurol.2017.0403.

[5] Bracke P., W. Christiaens and N. Wauterickx (2008), "The pivotal role of women in informal care", Journal of Family Issues, 29 (10), 1348-1378.

[6] Canta, C. and H. Cremer (2017), "Long-term care policy with nonlinear strategic bequests", TSE WP.

[7] Charles, S. and F. Carmichael, (1998), "The labour market costs of community care", Journal of Health Economics, 17, 747-765.

[8] Charles, K.K. and P. Sevak, (2005), "Can family caregiving substitute for nursing home care", Journal of Health Economics, 24, 1174-1190. 
[9] Cremer, H., P. Pestieau, and G. Ponthière, (2012), The economics of longterm care: a survey, Nordic Economic Policy Review, 2, 107-148.

[10] Fortin, N.M. (2005), "Gender role attitudes and the labour-market outcomes of women across OECD countries," Oxford Review of Economic Policy, 21 (3), 416-438.

[11] Haberkern, K. and M. Szydlik (2010), "State care provision, societal opinion and children's care of older parents in 11 European countries", Ageing and Society, 30, 299-323.

[12] Jakobsson, N., A. Kotsadam, A. Syse and H. Oien, (2016), Gender bias in public long-term care? A survey experiment aming care managers, Journal of Economic Behavior \& Organization, 131, 126-138.

[13] Klimaviciute, J. (2015), "Long-term care and myopic couples", mimeo.

[14] Konrad, K., H. Kühnemund, K. Lommerud and J. Robledo (2002), "Geography of the family", American Economic Review, 92, 981-997.

[15] Kotsadam, A. (2011), "Does informal eldercare impede women's employment? The case of european welfare states", Feminist Economics, 17 (2), 121-144.

[16] Norton, E.C. (2000), "Long-term care", in A. Cuyler \& J. Newhouse (Eds.): Handbook of Health Economics, Volume 1b, Chapter 17.

[17] O'Neill, J. (2000), "The gender gap in wages, circa 2000", The American Economic Review, 93 (2), 309-314.

[18] Schmid, T., M. Brandt and K. Haberkern (2012), "Gendered support to older parents: do welfare states matter?", European Journal of Ageing, 9, 39-50. 
[19] Stabile, M., A. Laporte and P.C. Coyte (2006), "Household responses to public home care programs", Journal of Health Economics, 25, 674-701.

[20] Tolkacheva, N., M.B. van Groenou and T. van Tilburg (2014), "Sibling similarities and sharing the care of older parents", Journal of Family Issues, 35 (3), 312-330.

[21] Van Houtven, C.H and E.C. Norton (2004), "Informal care and health care use of older adults", Journal of Health Economics, 23, 1159-1180. 


\section{Appendix}

\section{A First-best allocation}

Informal care. The FOC of (7) with respect to $a_{d}$ is

$$
\begin{aligned}
& \frac{1}{2} F\left(\hat{c}_{s d}\right)\left[p-\alpha w-I \gamma\left(\frac{1}{2} F\left(\hat{c}_{s d}\right)-1\right)+u^{\prime}\left(a_{d}\right)-\beta u^{\prime}\left(1-a_{d}\right)\right]-\frac{1}{2}\left[1-F\left(\hat{c}_{s d}\right)\right] \gamma \frac{1}{2} F\left(\hat{c}_{s d}\right) \\
& -\frac{1}{4} F\left(\hat{c}_{d d}\right) I \gamma \frac{1}{2} F\left(\hat{c}_{s d}\right)-\frac{1}{4} F\left(\hat{c}_{d d}\right) \gamma \frac{1}{2} F\left(\hat{c}_{s d}\right)-\frac{1}{4}\left[1-F\left(\hat{c}_{d d}\right)\right] 2 \gamma \frac{1}{2} F\left(\hat{c}_{s d}\right)=0 \\
\Leftrightarrow & \alpha w-p+\varphi+\left[\frac{1}{2} F\left(\hat{c}_{s d}^{F B}\right)+\frac{1}{4} F\left(\hat{c}_{d d}^{F B}\right)-1\right] I \gamma=u^{\prime}\left(a_{d}^{F B}\right)-\beta u^{\prime}\left(1-a_{d}^{F B}\right)
\end{aligned}
$$

where $\varphi$ in (A1) is obtained by collecting all terms multiplied by $\gamma$. The expression and the economic interpretation of $\varphi$ are in the main text (see expression (10) and comments below).

The FOC of (7) with respect to $a_{d 1}$ is

$$
\begin{aligned}
& \frac{1}{4} F\left(\hat{c}_{d d}\right)\left[p-\alpha w-I \gamma\left(\frac{1}{4} F\left(\hat{c}_{d d}\right)-1\right)-\gamma \frac{1}{4} F\left(\hat{c}_{d d}\right)+u^{\prime}\left(a_{d 1}\right)-\beta u^{\prime}\left(1-a_{d 1}\right)\right]-\frac{1}{4} F\left(\hat{c}_{d d}\right) \gamma \frac{1}{4} F\left(\hat{c}_{d d}\right) \\
& -\frac{1}{4}\left[1-F\left(\hat{c}_{d d}\right)\right] 2 \gamma \frac{1}{4} F\left(\hat{c}_{d d}\right)-\frac{1}{2} F\left(\hat{c}_{s d}\right) I \gamma \frac{1}{4} F\left(\hat{c}_{d d}\right)-\frac{1}{2}\left[1-F\left(\hat{c}_{s d}\right)\right] \gamma \frac{1}{4} F\left(\hat{c}_{d d}\right)=0 \\
\Leftrightarrow & \alpha w-p+\varphi+\left[\frac{1}{2} F\left(\hat{c}_{s d}^{F B}\right)+\frac{1}{4} F\left(\hat{c}_{d d}^{F B}\right)-1\right] I \gamma=u^{\prime}\left(a_{d 1}^{F B}\right)-\beta u^{\prime}\left(1-a_{d 1}^{F B}\right)
\end{aligned}
$$

where $\varphi$ in (A2) is again obtained by collecting all terms multiplied by $\gamma$.

Comparison of (A1) and (A2) reveals that $a_{d}^{F B}=a_{d 1}^{F B}$.

Finally, the FOC with respect to $a_{s 1}$ is

$$
\begin{aligned}
& \frac{1}{4} F\left(\hat{c}_{s s}\right)\left[p-w+u^{\prime}\left(a_{s 1}\right)-\beta u^{\prime}\left(1-a_{s 1}\right)\right]-\frac{1}{4}\left[1-F\left(\hat{c}_{d d}\right)\right] 2 \gamma \frac{1}{4} F\left(\hat{c}_{s s}\right)-\frac{1}{2}\left[1-F\left(\hat{c}_{s d}\right)\right] \gamma \frac{1}{4} F\left(\hat{c}_{s s}\right) \\
& -\frac{1}{2} F\left(\hat{c}_{s d}\right) I \gamma \frac{1}{4} F\left(\hat{c}_{s s}\right)-\frac{1}{4} F\left(\hat{c}_{d d}\right) I \gamma \frac{1}{4} F\left(\hat{c}_{s s}\right)-\frac{1}{4} F\left(\hat{c}_{d d}\right) \gamma \frac{1}{4} F\left(\hat{c}_{s s}\right)=0 \\
\Leftrightarrow & w-p+\varphi+\left[\frac{1}{2} F\left(\hat{c}_{s d}^{F B}\right)+\frac{1}{4} F\left(\hat{c}_{d d}^{F B}\right)\right] I \gamma=u^{\prime}\left(a_{s 1}^{F B}\right)-\beta u^{\prime}\left(1-a_{s 1}^{F B}\right)
\end{aligned}
$$

Once again $\varphi$ is obtained by collecting all terms multiplied by $\gamma$. Because of the gender wage gap we have $a_{s 1}^{F B} \equiv a_{s}^{F B}<a_{d}^{F B}$ so that $a_{s}^{F B}<\bar{a}^{F B}<a_{d}^{F B}$ and $I=0$. Hence, the first-best levels reduce to equations (8) and (9) in the main text. 
Critical costs. Let us now turn to the FOCs with respect to the critical cost levels. Since the procedure is similar for all FOCs we restrict ourselves to the FOC with respect to $\hat{c}_{s d}$. It is given by

$$
\begin{gathered}
\frac{1}{2} \hat{W}_{s d}^{1} f\left(\hat{c}_{s d}\right)-\frac{1}{2} W_{s d}^{0} f\left(\hat{c}_{s d}\right)-\frac{1}{2}\left[1-F\left(\hat{c}_{s d}\right)\right] \gamma \frac{\partial \bar{a}^{F B}}{\partial \hat{c}_{s d}} \\
-\frac{1}{4} F\left(\hat{c}_{d d}\right) \gamma \frac{\partial \bar{a}^{F B}}{\partial \hat{c}_{s d}}-\frac{1}{4}\left[1-F\left(\hat{c}_{d d}\right)\right] 2 \gamma \frac{\partial \bar{a}^{F B}}{\partial \hat{c}_{s d}}=0 .
\end{gathered}
$$

We have $\partial \bar{a}^{F B} / \partial \hat{c}_{s d}=(1 / 2) f\left(\hat{c}_{s d}\right) a_{d}^{F B}$ so that the above equation reduces to

$$
\hat{W}_{s d}^{1}-W_{s d}^{0}-\frac{1}{2}\left[1-F\left(\hat{c}_{s d}\right)\right] \gamma a_{d}^{F B}-\frac{1}{4} F\left(\hat{c}_{d d}\right) \gamma a_{d}^{F B}-\frac{1}{2}\left[1-F\left(\hat{c}_{d d}\right)\right] \gamma a_{d}^{F B}=0 .
$$

Inserting the welfare levels, we can write

$$
(p-\alpha w) a_{d}^{F B}-\hat{c}_{s d}^{F B}-\gamma \bar{a}^{F B}+u\left(a_{d}^{F B}\right)+\beta\left[u\left(1-a_{d}^{F B}\right)-u(1)\right]-\varphi a_{d}^{F B}=0 .
$$

Solving for $\hat{c}_{s d}^{F B}$ yields expression (11) in the main text.

\section{B FB implementation}

Informal care. The laissez-faire informal care provision by daughters in type $(s, d)$ and $(d, d)$ families is characterized by

$$
\alpha w-p=\Delta\left[u^{\prime}\left(a_{d}^{*}\right)-\beta u^{\prime}\left(1-a_{d}^{*}\right)\right]
$$

with a subsidy $\sigma_{d}$ on formal home care this becomes

$$
\alpha w-p+\sigma_{d}=\Delta\left[u^{\prime}\left(a_{d}^{*}\right)-\beta u^{\prime}\left(1-a_{d}^{*}\right)\right] \quad \Leftrightarrow \quad \Delta^{-1}\left[\alpha w-p+\sigma_{d}\right]=u^{\prime}\left(a_{d}^{*}\right)-\beta u^{\prime}\left(1-a_{d}^{*}\right) .
$$

The FB is given by

$$
\alpha w-p+\varphi=u^{\prime}\left(a_{d}^{F B}\right)-\beta u^{\prime}\left(1-a_{d}^{F B}\right)
$$

Implementing the $\mathrm{FB} a_{d}$ then requires

$$
\Delta^{-1}\left[\alpha w-p+\sigma_{d}\right]=\alpha w-p+\varphi \quad \Leftrightarrow \quad \sigma_{d}=(\Delta-1)[\alpha w-p]+\Delta \varphi .
$$


For $\alpha=1$, we have the subsidy for sons.

Critical costs. The laissez-faire informal care provision by daughters in type $(s, d)$ and $(d, d)$ families is characterized by

$$
\hat{c}_{s d}^{*}=\hat{c}_{d d}^{*} \equiv(p-\alpha w) a_{d}^{*}+\gamma \bar{a}+\Delta\left[u\left(a_{d}^{*}\right)+\beta\left[u\left(1-a_{d}^{*}\right)-u(1)\right]\right]
$$

with subsidies $\sigma_{d}$ and $\sigma_{s}$ on formal home care and a subsidy $\theta_{d}$ on the nursing home this becomes

$$
\hat{c}_{s d}^{*}=\hat{c}_{d d}^{*} \equiv\left(p-\sigma_{d}-\alpha w\right) a_{d}^{F B}-\theta_{d}+\gamma \bar{a}^{F B}+\Delta\left[u\left(a_{d}^{F B}\right)+\beta\left[u\left(1-a_{d}^{F B}\right)-u(1)\right]\right]
$$

The FB is given by

$$
\hat{c}_{s d}^{F B}=\hat{c}_{d d}^{F B} \equiv(p-\alpha w) a_{d}^{F B}+\gamma \bar{a}^{F B}+u\left(a_{d}^{F B}\right)+\beta\left[u\left(1-a_{d}^{F B}\right)-u(1)\right]-\varphi a_{d}^{F B}
$$

Implementing the $\mathrm{FB} \hat{c}_{d d}$ then requires

$$
\begin{aligned}
& \left(p-\sigma_{d}-\alpha w\right) a_{d}^{F B}-\theta_{d}+\gamma \bar{a}^{F B}+\Delta\left[u\left(a_{d}^{F B}\right)+\beta\left[u\left(1-a_{d}^{F B}\right)-u(1)\right]\right] \\
= & (p-\alpha w) a_{d}^{F B}+\gamma \bar{a}^{F B}+u\left(a_{d}^{F B}\right)+\beta\left[u\left(1-a_{d}^{F B}\right)-u(1)\right]-\varphi a_{d}^{F B} \\
\Leftrightarrow & \theta_{d}=\left(1-a_{d}^{F B}\right) \sigma_{d}+\varphi a_{d}^{F B}+(\Delta-1)\left[u\left(a_{d}^{F B}\right)+\beta\left[u\left(1-a_{d}^{F B}\right)-u(1)\right]\right]
\end{aligned}
$$




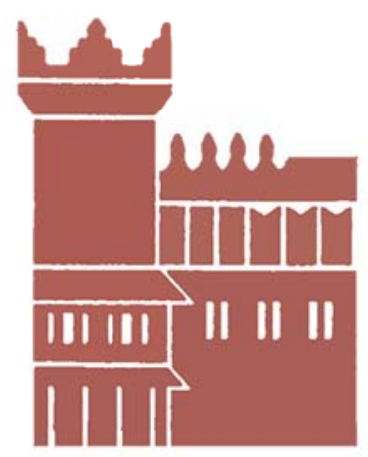

Alma Mater Studiorum - Università di Bologna DEPARTMENT OF ECONOMICS

Strada Maggiore 45

40125 Bologna - Italy

Tel. +39051 2092604

Fax +390512092664

http://www.dse.unibo.it 